Rev. Elev. Méd. Vét. Poys Trop. 1964, 17, 3, (369-375)

\title{
Elimination de l'acide pyruvique des milieux de culture en vue de favoriser la survie de Trypanosoma evansi
}

\author{
BALIS ( $j$.) \\ (Laboratoire de Farcha, Fort-Lamy Tchad)
}

\section{RÉSUMÉ}

L'acide pyruvique est le principal déchet du métabolısme glucidique de T. evansi.

L'auteur a d'abord étudié l'importance et la rapidité de sa formation puis sa toxicité pour Trypanosoma evansi et pense que son accumulation dans ie milieu constifue un obstacle majeur à la culture « in vitro».

Le présent travail a surtout pour objel l'étude de différents moyens d'ordres physique, chimique et biologique, destinés à élıminer l'acide pyruvique au fur et ò mesure de sa formation.

Les moyens physiques expérimentés sont les suivants :

o) La diffusion en gélose, c'est-à-dire l'utilisation des milieux diphasiques.

b) La diffusion à travers une membrane semi-perméable.

c) Le lavage contınu sur une membrane filtrante.

d) L'utilisation des propriétés adsorbantes des charbons.

Ces différentes techniques permetlent d"obtenir des résultats intéressants mais il est nécessaire de tenir compte du fall que des facteurs de croissance peuvent également être éliminés.

Il est possıble de neutraliser chimiquement l'acide pyruvique par l'hydroxylamine, le sulfite et le bisulfite de sodium, mais les composés formés présentent une toxicité non négligeable.

Enfin, biologiquement, on devrait pouvoir compléter l'appareıl enzymalique de Trypanosoma evansi et obtenir une dégradation complète de l'acide pyruvique.

2 iableaux.

Bıbliographie : 9 références.'

Trypanosomo evonsi, que l'on trouve dans le sang circulant d'un animal trypanosomé, y puise les éléments nécessaıres à sa vie et à sa multiplication. Les conditions semblent particulièrement favorables chez le rat où nous avons parfois constaté la présence de plus d'un million de parasites par $\mathrm{mm}^{3}$ de sang.

On pouvait 'penser que sa culture « in vitro» serait simple mais pourtant elle n'a jamais été réalisée. On s'aperçolt, en effet que cette culture est sous la dépendance de multıples facteurs, le plus souvent méconnus, et très liés les uns aux autres, c'est-à-dire que la présence ou l'absence de l'un d'eux suffit à rendre impossible toufe réussite.

Une des couses d'échec est la formation de 
déchets et spécialement ceux du catabolisme du glucose, que le flagellé a la possıbilıté d'éliminer lorsqu'il se multiplie dans le sang circulant.

KLIGLER, GEIGER et COMAROFF (5) parlent de l'acide lactique. CHRISTOPHERS et FULTON (4) notent une action nocive de l'acidification sur T. rhodesiense. KRIJGSMAN (6) pense que le principal déchet du métabolisme de $T$. evonsi est l'acide pyruvique. C'est aussi l'avis de MARSHALL (7) qui le prouve et trouve par des mesures précises que chez $T$. evonsi, les $7 / 8 \mathrm{e}$ du glucose utilisé sont transformés en acide pyruvique qui s'accumule dans le milieu.

Le but de notre travail a été de rechercher en premier lieu quelles étaient l'importance et la rapidité de formation de l'acide pyruvique, puis, après avoir mis en évidence sa toxicité pour $T$. evansi nous avons étudié et expérimenté différents moyens destinés à élımıner cet acide du milieu de culture.

\section{MATÉRIEL ET MÉTHODES}

La souche de $T$. evansı a été prélevée en 1961 sur un âne de Fort-Lamy.

Les différentes expérimentations ont été effectuées soit en milieux liquides, soit en milieux diphasıques :

- Milieux liquides : ils sont de 2 types.

1) Milieu au sang de cheval dont la composition est la suivante :

sang de cheval ........... $10 \mathrm{ml}$

liquolde « Roche $» . . . . . .1 \mathrm{ml}$

eau distillée ............. $90 \mathrm{ml}$

Hémolyse pendant 1 heure à la température du laboratoire.

phosphate bipotassique ...... $\quad 1 \mathrm{~g}$
glucose ...............
phosphate monopotassique :
Q. S. pour avoir $\mathrm{gH}$ 7.4.

Filtration sur papier puis sur Seitz.

2) Milieu synthétique de formule suivante :

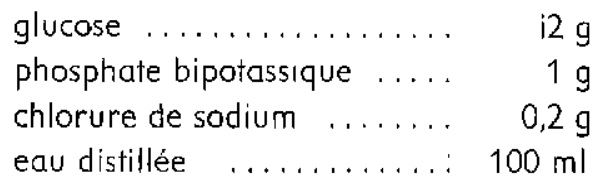

phosphate monopotassique :

Q. S. pour avoir $\mathrm{pH} 7,4$.

Filtration sur Seitz.

Milieux diphasiques : ils sont réalisés en tubes à essais avec comme phase liquide l'un des milieux précédemment décrits et comme phase solide de la gélose physiologique à 2 p. 100 dans laquelle est incorporée la substance détoxifiante.

Les dosages d'acide pyruvique ont été effectués par la technique de CARON et RAQUET (2) modifiée par nous-mêmes (1).

Toutes les expérımentations ont été réalisées dans une pièce climatisée maintenue automatiquement à la température de $25^{\circ}$.

\section{IMPORTANCE ET RAPIDITÉ DE FORMATION DE L'ACIDE PYRUVIQUE}

Nous avons procédé de la façon suivante : $200 \mathrm{ml}$ de milieu au sang sont ensemencés avec du sang de rat fortement parasité. Toutes les $30 \mathrm{mn}$ on prélève $6 \mathrm{ml}$ du mélange afin de pratıquer un dosage d'acide pyruvique et une numération des trypanosomes.

Le tableau $n^{\mathbf{0}} 1$ montre que l'acide pyruvique est nettement décelable dans le milieu dès la première demi-heure. Son taux croît presque linéairement en même temps que la quantıté de flagellés diminue. II semble exister un rapport entre ces deux phénomènes et nous avons été amenés à étudier la toxicité de l'acide pyruvique.

\section{TOXICITÉ DE L'ACIDE PYRUVIQUE POUR $T$, EVANSI}

Cette expérimentation a été faite sur milieux diphasiques avec comme phase solide de la gélose physiologique servant de support à l'acide pyruvique ou au pyruvate de Sodium, et comme phase lıquide, le milieu synthétıque.

Ce dernier est ensemencé en bloc, puis réparti dans les différentes séries de tubes à essais. En débuł d'expériences, nous avions 40.000 flagellés au $\mathrm{mm}^{3}$.

Le tableau no 2 nous donne les résultats relevés après $20 \mathrm{~h}$ d'incubation.

L'acide pyruvique est nettement plus toxique que son sel de sodium et une dose de $10 \mathrm{mg}$ pour $120 \mathrm{ml}$ (phase liquide et phase solide) soit $0,08 \mathrm{mg}$ 
TABLEAU NO I

\begin{tabular}{|c|c|c|}
\hline $\begin{array}{r}\text { Temps en } \\
\text { minutes }\end{array}$ & $\begin{array}{c}\text { Quantité de } \\
\text { pyruvate en } \\
\text { ug. par cm3 }\end{array}$ & $\begin{array}{r}\text { Numération des } \\
\text { trypenosomes }\end{array}$ \\
\hline 0 & 0 & 17.600 \\
30 & 0,03 & 17.000 \\
60 & 0,06 & 15.600 \\
90 & 0,09 & 14.000 \\
120 & 0,11 & 12.800 \\
150 & 0,14 & 11.000 \\
180 & 0,16 & 10.000 \\
210 & 0,18 & 8.000 \\
\hline
\end{tabular}

par ml est très nocive. Or, sı nous nous reportons au tableau no 1 , nous constatons que ce taux est atteint avant 90 minutes.

Dès la première heure, un milieu liquide peut donc être considéré comme impropre à toute culture de $T$. evansı, même s'il contient les facteurs de croissance nécessaires. Il est donc évident que si l'on veut avoir quelques chances de succès, Il faut en premier lıeu éliminer l'acide pyruvique du milieu.
Nous avons étudié un certain nombre de procédés (physiques, chimiques et brologıques) qui nous ont permis d'apporter un début 'de solution à ce problème,

Moyens physiques:

\section{1) Diffusion dans la gélose :}

C'est le procédé classiquement employé lorsqu'on cultive les trypanosomes en milieux diphasiques. La phase solide est toujours beaucoup plus volumineuse que la phase liquide ef l'acide pyruvique formé diffuse dans la gélose.

Une amélioration du rendement peutêtre obtenue en diminuant la température d'incubation. En effet, entre 25 et $370^{\circ} \mathrm{Cl}$ 'intensité du métabolisme de $T$. evansi obéit à la loi de VAN't HOFF, c'està-dıre qu'elle est divisée par 2,5 quand la température baisse de $10^{\circ} \mathrm{C}$. Par contre, la vitesse de diffusion étant proportionnelle à la température absolve sera, pour le même intervalle, divisée par 1,03. Il est donc possible de diminuer fortement la production d'acide pyruvique tout en né changeant pratıquement rien d̀ son 'élimination.

TABLAAU No II

\begin{tabular}{|c|c|c|c|}
\hline Phose liquide & Phase solide & Nombre de tubes & $\begin{array}{l}\text { Nombre de tubes } \\
\text { par mm3 après } 20 \mathrm{~h} \text {. }\end{array}$ \\
\hline \multirow{5}{*}{$\begin{array}{c}\text { Milieu } \\
\text { synthétique } \\
\text { et } \\
\text { sang de } \\
\text { rat parasité } \\
2 \text { ml. par } \\
\text { tube. }\end{array}$} & $\begin{array}{l}\text { gélose physiologique } \\
100 \mathrm{ml} .\end{array}$ & 10 & 900 \\
\hline & $\begin{array}{l}\text { gélose physiologique } \\
\text { t00 ml + } 100 \mathrm{mg} \\
\text { d'acide pyruvique }\end{array}$ & 10 & 0 \\
\hline & $\begin{array}{l}\text { gélose physiologique } \\
100 \mathrm{ml}+10 \mathrm{mg} . \\
\text { d'acide pyruvique }\end{array}$ & 10 & 440 \\
\hline & $\begin{array}{l}\text { gélose physiologique } \\
100 \mathrm{ml.t} 100 \mathrm{mg} \text {. } \\
\text { pyruvate de } \mathrm{Na}\end{array}$ & 10 & 390 \\
\hline & $\begin{array}{l}\text { gélose physlologlque } \\
100 \mathrm{ml} .+10 \mathrm{mg} . \\
\text { pyruvate de } \mathrm{Na}\end{array}$ & 10 & 610 \\
\hline
\end{tabular}


Les cultures de trypanosomes pathogènes « in vitro » ne réussissent d'ailleurs qu'aux envırons de $25^{\circ}$.

\section{2) Diffusion à travers une membrane semi- perméable :}

Une membrane seml-perméable laisse passer les cristalloides et doit donc permettre une élimination correcte de l'acide pyruvique. Afin de vérifier cette hypothèse, nous avons réalisé le dispositif suivant :

Un sac de cellulose contenant $22 \mathrm{ml}$ de milieu au sang ( $A$ ) ensemencé avec $T$. evansi est suspendu dans une fiole d'ERLENMEYER et baigne dans $133 \mathrm{ml}$ de milıeu synthétique (B). $22 \mathrm{mli}$ de milieu au sang, ensemencé, sont gardés comme témoin (C).

Au début de l'expérience, nous avions en $A$ et $C, 27.000$ flagellés au $\mathrm{mm}^{3}$. Après $20 \mathrm{~h}$ d'incubation nous avons effectué une numération des trypanosomes sur $A$ et $C$, puis un dosage d'acide pyruvique sur $A, B$ et $C$, nous avons noté les résultats suivants :

Numération des trypanosomes :

$$
\begin{aligned}
& \text { A }: 9.200 \\
& C: 15.600
\end{aligned}
$$

Dosage de l'acide pyruvique :

A : $0,08 \mathrm{mg} / \mathrm{ml}$ soit au total $0,08 \times 22=1,76 \mathrm{mg}$ B: $0,03 \mathrm{mg} / \mathrm{ml}$ solt au total $0,03 \times 1334 \mathrm{mg}$ C : $0,30 \mathrm{mg} / \mathrm{ml}$ soit au total $0,30 \times 22=6,6 \mathrm{mg}$.

L'acide pyruvique a donc bien diffusé en $B$ mais l'équilıbre n'a pas été atteint puisque la quantité au $\mathrm{ml}$ est supérieure en $\mathrm{A}$. Par contre, en C l'acide s'est accumulé et le taux en est beaucoup plus important.

Il est certain qu'un dispositif ayant un rapport masse/surface très petıt doit permettre d'atteındre des résultats très corrects.

Puisque le principal déchet se trouve en partie élıminé, nous devrions trouver le plus de trypanosomes en $A$ et la quantité d'acide pyruvique contenue en $A$ et $B$ devrait être supérieure à celle trouvée en $C$. Or, c'est l'inverse que nous observons. On est donc amené à penser qu'un facteur favorable a diffusé de $A$ vers $B$ et il est nécessaire d'en tenir compte dans tout système utilisant la dialyse comme moyen détoxifiant.

\section{3) Lavage continu :}

Cette méthode doit en principe permettre l'élımination totale de l'acide pyruvique. Nous l'avons expérımentée à l'aide du disposıtif suivant :

On verse quelques $\mathrm{ml}$ de milieu au sang contenant des trypanosomes dans un petit filtre Seitz, puis on ferme sa partile supérieure par un bouchon. Ce dernier est traversé par un comptegouttes auquel est adapté un tuyau souple amenant du milieu au sang sous une faible pression ( 40 à $50 \mathrm{~cm}$ d'eau). Le liquide to me goutte à goutte dans le filtre, comprime l'air qui s'y trouve et une fois l'équilibre atteint, il passe à travers le disque d'amiante cutant de liquide qu'il en rentre à la partıe supérieure.

Les trypanosomes sont donc en suspension dans un milieu continuellement renouvelé. L'expérience montre que l'acide pyruvique est bien éliminé ; il n'en reste pas dans le filtre et on n'en trouve que des traces dans le liquide de perfusion. Mais alors qu'au moment de l'ensemencement on dénombrait 4.600 flagellés au $\mathrm{mm}^{3}$, on n'en retrouve $20 \mathrm{~h}$ après que 1.800 dans le témoin et mons de 10 dans le filtre. II semble donc y avoir eu privation d'un ou de plusieurs facteurs nécessaires provenant de $T$. evansi luimême. L'un d'eux est peut être analogue à l'exoantıgène signalé par WEITZ (8-9) chez 1. brucei.

En résumé, la technique du lavage continu permet une élimination très correcte de l'acide pyruvique et des autres déchets de métabolisme mais ne peut être envisagée qu'avec un milieu contenant tous les facteurs nécessaires y compris ceux provenant de $T$. evansi.

\section{4) Utilisation des propriétés adsorbantes des} charbons :

Le charbon de bois et le noir animal sont capables à des degrés différents d'adsorber des gaz, des vapeurs et de nombreuses substances minérales ou organiques en solution. Ils se comportent comme une sorte d'éponge présentant une surface interne considérable et leur pouvoir absorbant est fonction de leur état de division.

Le charbon végétal est loin d'être un carbone pur. Outre quelques gaz, il contient 1 à 8 p. 100 de cendres à réaction alcaline.

Le charbon animal purifié possède un pouvoir adsorbant beaucoup plus grand et, en dehors 
du carbone à l'état très divisé, on ne trouve que des substances sans affinités chimiques comme la silice.

Nous avons constaté que surtout le noir animal purifié était capable d'adsorber de petites quantités d'acide pyruvique.

L'influence des charbons sur la survie de $T$. evonsı a été recherchée par la technıque des milieux diphasıques; le norr animal est inclus dans la gélose ef la phase liquide est du milieu au sang.

Pour 20.000 flagellés au $\mathrm{mm}^{3}$ en début d'expérience nous avons trouvé après 20 heures d'incubation les résultats suivonts :

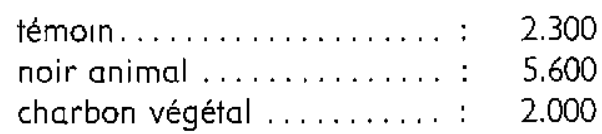

L'influence favorable du noir animal paraît assez nette ei comme cette substance est chimiquement inactive, seules ses propriétés physiques sont en cause.

Ces résultats s'expliquent si on considère que sont éliminés en priorité les corps de faible poids moléculaire qui diffusent rapidement dans la gélose. Malheureusement, d'autres substances favorables subıssent le même sort ef les résultats sont différents si on mélange directement le charbon animal au milieu liquide.

En effet, nous avons ensemencé avec la même quantité de T. evansi 3 fioles d'ERLENMEYER : A - B - C, contenant :

A : $50 \mathrm{ml}$ de milieu au sang $+1 \mathrm{~g}$ de charbon animal

B : $50 \mathrm{ml}$ de milieu au sang traité par $1 \mathrm{~g}$ de charbon animal puis filtré sur Seitz

$C: 50 \mathrm{ml}$ de milieu au sang.

Pour 10.000 trypanosomes au départ de l'expérience, nous avons retrouvé après 20 heures d'incubation :

$$
\begin{aligned}
& \text { A : } 4.900 \\
& \text { B : } 6.100 \\
& \text { C : } 6.700
\end{aligned}
$$

Le nombre le plus élevé correspond au témoin $C$ et en $A$, il y a eu adsorbtion d'une partie de l'acide pyruvique mais également de facteurs nécessaires de gros poids moléculaire, provenant du flagellé ou du sang de rat. Ces facteurs se trouvent également dans le milieu au sang, mais en très faıble quantité puisqu'uri traitement au noir animal suivi de filtration ne modifie que peu la survie.

L'utilisation des charbons ne résoud donc pas correctement le problème, cependant une étude, en fonction du $\mathrm{pH}$, des propriétés adsorbantes d'cutres substances peut à notre avis, aboutır à des résultats intéressants.

\section{Moyens chimiques :}

1) Hydroxylomine.

Au cours de diverses expérimentations, nous avons constaté l'effet favorable de substances telles que la Colamine ou l'ammonioque à très faible dose (1 pour 10.000) et nous avons été amenés à effectuer des essals avec l'hydroxylamine.

Des dosages systématiques de l'acide pyruvique nous ont permis d'observer qu'il étalt partiellement éliminé par l'hydroxylamine.

Chımiquement, une molécule de sulfate se combine à deux molécules d'acide pyruvique.

Expérimentalement, le sulfate d'hydroxylamine à la dose de $10 \mathrm{mg}$ pour $100 \mathrm{ml}$ de milieu améliore la survie mais présente cependant une certaine toxicité. Cette dernière peut être rédulte si on neutralise l'acide sulfurique soit par de la soude, et dans ce cas on obtient un' mélange de sulfate de sodium et d'hydraxylamine, soit par traitement au carbonate de baryum en excès et filtration sous vide pour éliminer le gaz carbonique.

Les résultats sont comparables et dans les 2 cas, on peut quadrupler la dose d'hydroxylamine. Mais la toxicité du composé formé avec l'acide pyruvique, n'étant pas négligeable, ce procédé n'offre que des possibilités limitées.

2) Sulfite ef bisulfite de sodium :

Ces deux corps semblent se combiner à l'acide pyruvique dans des proportions qui ne sont pas définies.

Leur toxicité est nettement moins importante que celle de l'hydroxylamine puisqu'on peut en ajouter $100 \mathrm{mg}$ pour $100 \mathrm{ml}$ de milieu.

Leur action favorable sur la survie est surtout due, à notre avis, à leurs propriétés réductrices car des corps tels que la cystéine, la glutathion réduit ou la réductone ont le même effet. 


\section{Moyens biologrques:}

L'éłude de ces moyens relativement complexes, n'a été qu'ébauchée.

De nombreux microorganismes sont capables de proliférer dans un milieu synthétique, ne contenant que de l'azote minéral et du pyruvate de sodium. Ils élaborent rapidement de l'alanine et de l'acide glutamique. C'est le cas des germes suvants :

Proteus vulgaris, Pseudomonas aeruginosa, Salmonella paratyphi B (Césaire-Boiron - Kerharo et Attiso (3)),

D'autres germes sont complètement autotrophes et peuvent faire la synthèse de leurs acides amınés à partir de l'azote atmosphérique et de l'acide pyruvique. C'est le cas de l'Azotobacter présent dans le sol et que l'on isole facilement sur terre moulée additionnée de pyruvate de sodium.

Des essals de culture en symbiose avec T. evansi n'ont pas été concluants car l'Azotobacter: n'a pas une croissance très rapide ef n'absorbe de ce fait qu'une faible partie de l'acide pyruvique.

De plus, cultıvé dans un milieu contenant du glucose il utilise de préférence ce dernier et on ne retire donc aucun bénéfice de l'association.

A notre avis, il y a des chances de succès en utilisant un germe à croissance rapide tel que Proteus vulgaris à partir duquel on pourrait opérer une sélection sur milieu synthétique au pyruvate. En extrayant des corps microbiens les enzymes présidant au catabolisme de l'acide pyruvique ef en les ajoutant au milieu au sang, on complèterait en quelque sorte l'apparell enzymatique de $T$. evansi.

\section{CONCLUSION}

L'accumulation d'acide pyruvique dans le milieu constitue un obstacle majeur à la culture « in vitro» de $T$. evansı. II est donc nécessaire d'éliminer ce déchet de métabolisme et différents moyens d'ordre physique, chimique et biologique ont été expérimentés.

Dans l'état actuel de nos recherches, les moyens physiques nous semblent les plus efficoces et ont le grand avantage d'être dépourvus de toxicité. Cependant, ils permettent également l'élimination de facteurs non identifiés indispensables à la multiplication de $T$. evansi et on doit tenir compte de ce falt dans les réalisations technuques.

Une détoxication chimique est assez délicate à manier car en définitive on recule le problème en remplaçant une substance toxique par une autre qui l'est un peu moins.

En fin, les techniques bıologiques bien que théoriquement pleines de promesses nécessitent encore de longues recherches avant d'aboutir qu résultat désiré.

\section{SUMMARY}

The elimination of pyruvic acid from the medium in order to improve the culture of Tryponosomo evansi

Pyruvic acid is the principal waste product of the carbohydrate metabolism of $T$. evansi.

The author has first studied the quantity and the rapidity of its formation and then its toxicity for $T$, evansi and thinks that its accumulation in the medium constitutes a major obstacie to the culture «In vitro ».

The present work is intended particularly to study the different means physical, chemical and biological which may possibly eliminate pyruvic acid as soon as is produced.

The physical methods tried are the following :

a) Diffusion in gelose, that is the utilisation of diphasic media.

b) Diffusion across a semipermeable membrane.

c) Continuous washing on a filtering membrane.

d) Utilisation of absorbant products of coal.

These different techniques produce interesting results but one must bear in mind the fact that growth factors could also be eliminated.

It is passible to neutralise pyruvic acid chemically by hydroxylamine sodium sulphite, and sodium bisulphite but the substances formed are themselves to some extent toxic. 
Finally, from a biological aspect, it should be possible to complete the enzymalic cycle of $T$. evansi and obtain a complele degradation of pyruvic acid.

2 fobles.

9 references.

\section{RESUMEN}

Elimicación del ácido pirúvico en los medios de cultura para favorecer el desarollo de Trypanosoma evansi

El ácido pirúvico es el principal resto del metabolismo de los glucidos de T. evansi.

Desde luego el autor estudió la importancia y la rapidez de su formación, después su toxicidad en cuanta al Trypanosoma evansi y piensa que su acumulación en el medio constituye un obstáculo mayor por el cultivo « in vitro ».

El presente trabajo tiene sobretodo por objeto el estudio de las diferentes facultades fissicas, quimicas y biológicas, que eliminaran el ácido pirúvico a medida de su formación.

Los medios fisicos experimentados son las siguientes :

a) La difusión en gelosa, es decir la utilización de los medios difasicos.

b) La difusión a traves una membrana semi permeable.

c) El lavado continuo en una membrana filirante.

d) La utilización de las cualidades absorbentes de los carbones.

Se puede obtener, con estas diferentes técnicos resultados interesantes, pero es necesario tomar en consideración que se pueden también eliminar los factores de crecimiento.

Es posible neutralizar quimicamente el acido pirúvico con la hidróxilamina, el sulfita y el bisulfito de sodio, pero los compuestos químicos presentan una roxicidad apreciable.

Al fin, biologicamente, se deberia poder completar el aparato enzímático del Trypanosoma evansi y obłener una degradación compleia del ácido pirúvico

2 cuadros.

Biblıográfia : 9 referencias.

\section{BIBLIOGRAPHIE}

1. BALIS (J.). - Recherche ef dosage de l'acide pyruvique dans les liquides biologiques. Rev. Elev. Méd. vét. Poys trop., 1963, 16 (4).

2. CARON (H.) et RAQUET (D.). - Caractérisation ef dosage de l'acide pyruvique. Application à la recherche de l'acide lactique. j. P. - C 1942, t. 2, p. 333.

3. CESAIRE (O. G.), BOIRON (H.), KERHARO (J.) et ATISSO (M.). - Peut-on envisager la préparation de certains aminoacides par synthèse bactérienne? Af. méd., 1962, (5): 359.

4. CHRISTOPHERS (S. R.) et FUI.TON (J. D.). Observations on the respiratory metabolism of Malaria parasites and Trypanosomes. Ann. trop. Med. Parasit. 1938, $32:$ 43-75.

5. KLIGLER (I. J.), GEIGER (A.) ef COMA-
ROFF (R.). - Effeci of the nature and composition of the substrate on the developpment and Viability of Trypanosomes. Ann. trop. Med. Parasit., 1930, $24: 329$.

6. KRIJGSMAN (B. J.). - Vergleichend physiologische Untersuchungen über den Stoffwechsel von Trypanosoma evansi im Zusammenhang mit der Aupassung an Wirtstier. Z. vergl. Physiol., 1936, $23: 663$.

7. MARSHALL (P. B.). - The glucose metabolism of Trypanosoma evansi and the action of trypanocides. Brit. J. Pharmacol., 1948, 3: 8-14.

8. WEITZ (B.). - The properties of some antigens of Trypanosoma brucei. J. gen. Microbiol., 1960, $23: 589-600$.

9. WEITZ (B.). - A soluble protective antigen of Trypanosoma brucei. Nature, 1960, 185 : 788-89. 